\title{
ABSTRACTS OF PAPERS FROM OTHER JOURNALS
}

\author{
J. Craig Wheeler and A. G. W. Cameron: 'The Effect of Primordial Hydrogen/Helium Fractiona- \\ tion on the Solar Neutrino Flux', Astrophys. J. 196 (1975), 601.
}

If hydrogen and helium are immiscible below some critical temperature, gravitational separation could occur in the proto-Sun, resulting in a nearly pure helium core and a nearly pure hydrogen shell. We have constructed solar models according to this scenario and find the neutrino flux reduced to 1.5-3 SNU. (Authors' abstract.)

G. W. Cushman, L. Farwell, G. Godden, and W. A. Rense: 'Solar Line Profiles of He i $584 \AA$ and He II 304 \&', J. Geophys. Res. 80 (1975), 482.

A grazing incidence spectrograph launched in a Black Brant rocket August 30, 1973, at White Sands Missile Range obtained high spectral resolution data of the solar radiation between 200 and $700 \AA$. Absolute intensities and profiles of the brighter lines in this spectral region were measured. Results for the He I $584 \AA$ and He II $304 \AA$ lines are presented. For the He I $584 \AA$ line the full width at half intensity is $0.08 \AA$, and the intensity for the solar disk is $1.3 \times 10^{9}$ photon $\mathrm{cm}^{-2} \mathrm{~s}^{-1}$. For the $304 \AA$ He II line these values are $0.12 \AA$ and $6.3 \times 10^{9}$ photon $\mathrm{cm}^{-2} \mathrm{~s}^{-1}$, respectively.

L. D. DE FEITER

JACQues M. Beckers: 'A Search for Deuterium on the Sun', Astrophys. J. 195 (1975), L43.

From observations of the $\mathrm{H} \alpha$ line of deuterium in quiescent solar prominences, $\mathrm{I}$ have determined a new upper limit to the solar $\mathrm{D} / \mathrm{H}$ ratio of $2.5 \times 10^{-7}$. (Author's abstract.)

J. W. Harvey and M. Schwarzschild: 'Photoelectric Speckle Interferometry of the Solar Granulation', Astrophys. J. 196 (1975), 221.

Using the McMath solar telescope on a morning of average seeing it has been found possible, with the help of photoelectric speckle interferometry, to detect securely the existence of details in the quiet solar granulation up to a wavenumber of $240 \times 10^{-4} \mathrm{~km}^{-1}$, i.e., a wavelength of one-third of an arc second. (Authors' abstract.)

J. Kubota, T. Kureizum, and S. Koyama: 'The Asymmetry of Na I D Line Profiles at the Root of a Disk Surge', Publ. Astron. Soc. Japan 26 (1974), 495.

The velocity field in the bright knot at the foot-point of a surge was examined by using the line-profiles of $\mathrm{Na}$ I D1 and D2. The spectrogram used was taken after the ejection of the surge.

A quite remarkable asymmetry is found in the core part of Na I D2 line, while no such drastic asymmetry is seen in $\mathrm{Na} I \mathrm{D} 1$ line as well as in other lines like Al I lines. The asymmetry in D2 line indicates the existence of downward-moving material above the enhanced chromosphere with a maximum velocity of $3 \mathrm{~km} \mathrm{~s}^{-1}$. The continued presence of the falling material with the abovementioned velocity suggests that there should be a braking action in the high chromosphere on the falling material which has otherwise $50-250 \mathrm{~km} \mathrm{~s}^{-1}$ from the analysis of the material velocity in the same surge in the corona by Tamenaga et al. (1973).

Y. UCHIDA 
BRUno Coppr: 'Remarks on Sequence-of-Plasma-Instabilities Models of Solar Flares', Astrophys. J. 195 (1975), 545.

The main requirements of solar flare models that explain their development in terms of an appropriate sequence of plasma instabilities are discussed. This sequence is characterized by a 'preheating phase' of the local chromospheric plasma, a 'flash phase' resulting from an explosive instability leading to particle acceleration and onset of plasma turbulence, and in the case of large flares by a 'hot phase' in which a significant amount of magnetic energy is converted into plasma flow and thermal energy. The relevant evolution of the electron distribution function and the two-dimensional flow and magnetic field configurations that can be consistent with the above-mentioned model are considered. (Author's abstract.)

G. A. Doschek, U. Feldman, K. P. Dere, G. D. Sandlin, M. E. VanHoosier, G. E. Brueckner, J. D. Purcell, and R. TouseY: 'Forbidden Lines of Highly Ionized Iron in Solar Flare Spectra', Astrophys. J. 196 (1975), L83.

Forbidden lines of Fe XVIII, Fe XIX, and Fe Xxr are identified at 974, 1118, and $1354 \AA$, respectively, in NRL ATM solar flare spectra. These lines are due to magnetic dipole transitions between levels of the ground configurations. The widths of the Fe XIX and Fe XXI lines are $\sim 0.5 \AA$, which is substantially greater than expected in ionization equilibrium. The intensity-time behavior and the widths of these lines are discussed for the 1973 June 15 flare. (Authors' abstract.)

L. W. Acton, R. C. Catura, and E. G. Joki: 'Oxygen-to-Neon Abundance Ratio in the Solar Corona', Astrophys. J. 195 (1975), L93.

The oxygen-to-neon abundance ratio in the solar corona is determined to be $4.7 \pm 1.5$ on the basis of a relatively model-independent analysis utilizing 25 separate measurements of the $\mathrm{O}$ VIII to Ne IX resonance line ratio. The stated uncertainty includes random and possible systematic experimental error in the data but does not include possible error in the calculated flux ratios which have been fitted to the observations. This abundance ratio is smaller than most published results based upon EUV or $\mathrm{X}$-ray observations but is in good agreement with direct measurements of flare cosmic rays. (Authors' abstract.)

\section{Richard Fisher and Steven Musman: 'Detection of Coronal Holes from $\lambda 5303$ Fe xiv Observa- tions', Astrophys. J. 195 (1975), 801.}

We use photoelectric measurements of intensity to estimate the distribution of volume emissivity in the coronal green line ( $\lambda 5303 \mathrm{Fe}$ xIV) over the solar surface. Using this distribution and a calculated dependence of emissivity as a function of $T$ and $N_{e}$, it is possible to estimate a coronal density distribution. An example is given which shows a region of drastically reduced emissivity - a coronal hole which was simultaneously detected by spacecraft. (Authors' abstract.)

\section{H. S. Sawant, S. K. Alurkar, and R. V. Bhonsle: 'New Microstructure of Decametre Solar Radio Bursts', Nature 253 (1975), 329.}

The authors report a new type of solar decametric bursts from their high-resolution $(\approx 5 \mathrm{kHz}$ in frequency and $\approx 10 \mathrm{~ms}$ in time) spectral observations made over a frequency range from 34.5 to 35 $\mathrm{MHz}$. Each of these bursts is characterized by a pair of components which run parallel. The first component is essentially a fast drift comprising patches of strong and weak emissions (or emission gaps). After a short time delay (centered around 1-2 $\mathrm{s}$ or 10-15 s) the second component occurs with narrow emission bands whose frequency ranges correspond to the emission gaps in the first component. The authors call these bursts, therefore, 'complementary bursts'. Possible explanation is suggested. 
P. N. Swanson and R. Kuseski: 'The Solar Spectrum at 8 mm', Nature 253 (1975), 513.

The authors have measured the brightness temperature at 36.0 and $37.75 \mathrm{GHz}(\lambda \approx 8 \mathrm{~mm})$ and the slope of the brightness curve between the two frequencies, using the new moon as a calibration source. The half power beamwidth was $14^{\prime}$. The obtained brightness temperature of the Sun is $7920 \pm 90 \mathrm{~K}$ at $36 \mathrm{GHz}$ and $7510 \pm 130 \mathrm{~K}$ at $37.75 \mathrm{GHz}$ (with the Moon's brightness temperature of 186 and $185 \mathrm{~K}$, respectively) and the slope is found to be $1.07 \pm 0.3$.

O. NAMBA

J. E. Beckman, J. C. G. Lesurf, and J. Ross: 'Submillimetre Brightness Spike at the Solar Limb'. Nature 254 (1975), 38.

During the total solar eclipse of June 30, 1973 submillimetre observations (at $0.4,0.8$, and $1.2 \mathrm{~mm}$ ) have been made from the highflying Concorde 001, using a rapid-scanning Michelson interferometer. The brightness temperature distributions along the radius of the solar disk at the three wavelengths show a distinct brightness spike which extends from $5^{\prime \prime}$ inside to $4^{\prime \prime}$ outside the optical limb, suggesting that the spike might be due to emission from chromospheric spicules. (See the accompanying figure.)

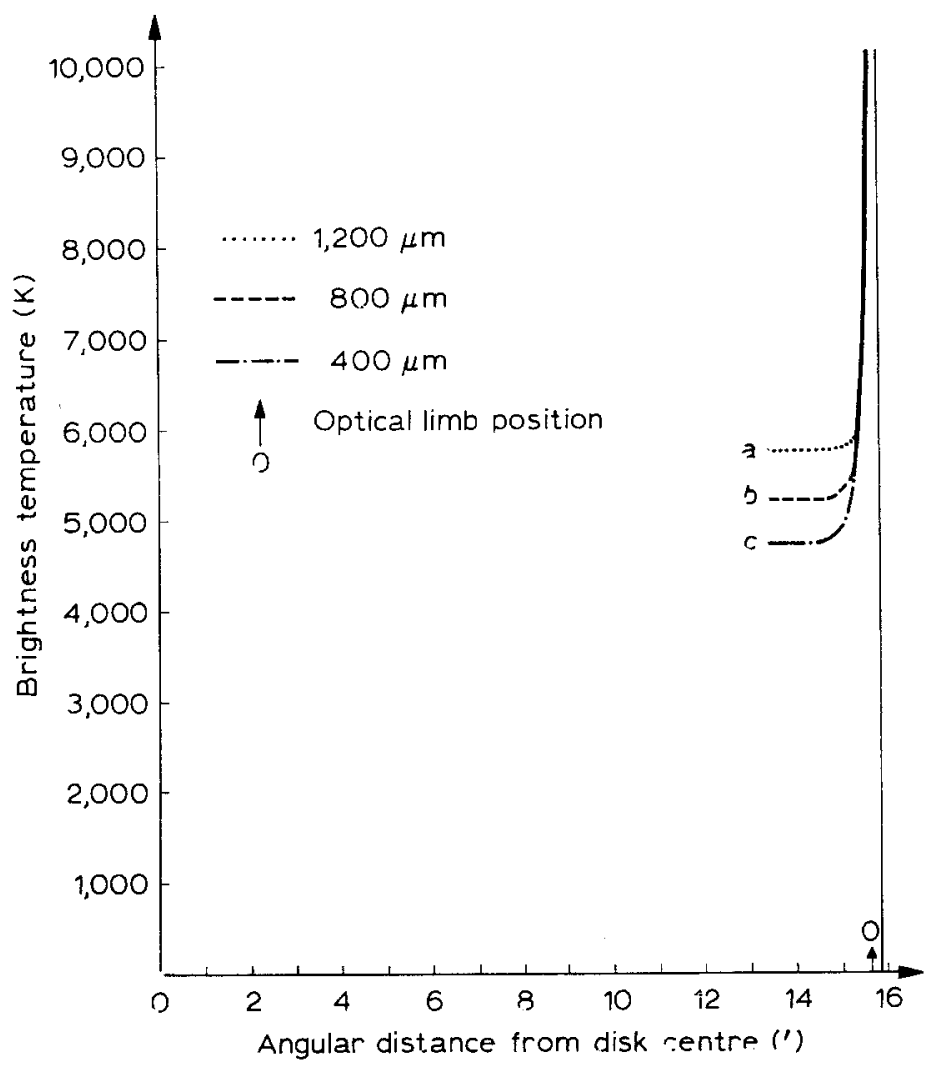

O. NAMBA 
K. KaI and H. NAKAJMa: 'Coronal Magnetic Fields Inferred from Source Distribution of Correlated Bursts and Concurrent Type I Storm', Publ. Astron. Soc. Japan 26 (1974), 376.

Numerous transient bursts (mostly type III) concurrent with a longlived type I noise storm were observed from 4 to 12, March 1972 with the Nobeyama $160 \mathrm{MHz}$ interferometer. The transient bursts occurred in many cases almost simultaneously or successively with a few second delay in separate sources with opposite polarity. These correlated bursts suggest that the separate sources must be linked by weak magnetic fields surrounding strong magnetic fields (presumably associated with the concurrent type I storm), having a common source of fast particles in a region lower than the $160 \mathrm{MHz}$ plasma level. Possible configurations of magnetic field above the associated active region are investigated on the basis of radio observations. (Authors' abstract.)

S. Cuperman and N. Metzler: 'Solution of Three-Fluid Model Equations with Anomalous Transport Coefficients for the Quiet Solar Wind', Astrophys. J. 196 (1975), 205.

A three-fluid model consisting of continuity, momentum, and energy equations is used to describe the average behavior of the electrons $(e)$, protons $(p)$, and $\alpha$-particles $(\alpha)$ in the quiet (undisturbed) expanding solar corona. Friction forces due to the possible occurrence of different $e, p$, and $\alpha$ streaming velocities are incorporated in the model equations. The transport coefficients are allowed to include (besides collisional interactions) phenomenological contributions from turbulent wave-particle interactions existing in the solar wind. The model assumes spherical symmetry and neglects such effects as viscosity, rotation, and magnetic fields. (However, the inhibition of the heat flux across the interplanetary magnetic field is implicitly taken into account in the expression used for the anomalous thermal conductivity.)

The numerical solution of the equations is achieved by using a perturbation-method procedure in which a two-fluid solution for electrons and protons is followed by a solution for the $\alpha$-particles.

These model-equations predict the following features for the $\alpha$-particles at $1 \mathrm{AU}$ : (i) equal $\alpha$ particle and proton streaming velocities, (ii) an $\alpha$-particle to proton temperature ratio of about 3.5 , and (iii) an $\alpha / p$ density ratio of about 0.035 (which is also less than half of that in the solar corona). These results explain the $\alpha$-particle characteristics observed at 1 AU. (Authors' abstract.)

Arild Gulbrandsen: 'The Solar $M$-Region Problem - An Old Problem Now Facing its Solution?', Planetary Space Sci. 23 (1975), 143.

The problem of the solar $M$-region (magnetic effective region) is reviewed and discussed. The discussion, based on analyses published on the $M$-region problem during the last $40 \mathrm{yr}$, leads to a conclusion that the $M$-regions should be identified with the central portion of magnetically open solar regions, or coronal holes.

O. NAMBA

\section{Space Science Reviews 17 (1975), 171.}

This part (the combined issue Nos. 2/3/4) of S.S.R. contains 16 review papers on the Sun, interplanetary medium, and the magnetosphere, presented at the International Solar-Terrestrial Physics Symposium held in São Paulo, Brazil, on June 17-24, 1974. These papers are listed below without abstract. Twelve review papers on Atmospheric Physics will appear in the Journal of Atmospheric and Terrestrial Physics in June 1975. Taken together, these papers form an up-to-date picture of solarterrestrial physics since the 1970 Symposium held in Leningrad.

List of papers: Introduction. - J. W. Dungey / Neutral Sheets. - L. D. de Feiter / Chromospheric Flares or Chromospheric Aurorae? - Tatsuzo Obayashi / Energy Build-Up and Release Mechanisms in Solar and Auroral Flares. - Takesi Nagata / Auroral Flares and Solar Flares. - Marcia Neugebauer / Large-Scale and Solar-Cycle Variations of the Solar Wind. - Heinrich J. Völk / Microstructure of the Solar Wind. - Murray Dryer/Interplanetary Shock Waves: Recent Developments. - L. F. Burlaga / Interplanetary Streams and Their Interaction with the Earth. - A. Nishida / Interplanetary 
Field Effect on the Magnetosphere. - Gregor Morfill / Study of Magnetospheric Dynamics Using Energetic Solar Particles. - Christopher T. Russell / The Third Solar Wind Conference: A Summary. - R. W. Fredricks / Wave-Particle Interactions and Their Relevance to Substorms. - Michael Schulz / Geomagnetically Trapped Radiation. - R. A. Wolf / Ionosphere-Magnetosphere Coupling. - P. A. Cloutier and H. R. Anderson / Observations of Birkeland Currents. - K. Schindler / Plasma Fields in the Magnetospheric Tail.

K. Kawabata, H. Ogawa, Y. Sofue, and I. Suzuki: 'The $35 \mathrm{GHz}$ Solar Interferometer at Nagoya', Publ. Astron. Soc. Japan 26 (1974), 387.

A description is given for the $35 \mathrm{GHz}$ grating interferometer recently constructed at the Department of Physics, Nagoya University. The instrument consists of eight paraboloidal antennae on Cassegrain mount, pointed downward, with steerable plane reflectors feeding the solar beam into them. This type of arrangement eliminates the effect of rain drops on the primary feed, and also the needs of rotary joints in the waveguide system. The interferometer span is $1913 \lambda$, $\mathrm{E}-\mathrm{W}$, and the angular resolution is $1.4^{\prime}$ at the meridian. The scanning time is about $50 \mathrm{~s}$ and the regular observation time is 7 to $10 \mathrm{hr}$ depending on the season. Some detail of the performance and examples of observational data are given.

Y. UCHIDA

M. STIx: 'Comments on the Solar Dynamo', Astron. Astrophys. 37 (1974), 121.

A variety of problems concerning the solar dynamo are studied: (1) The theories of Leighton and Krause and Steenbeck are compared in detail. (2) The effect of turbulent magnetic diffusion is illustrated and arguments against Piddington's criticism are given. (3) Longitude-dependent solutions of the dynamo equations are investigated in the context of the sector structure of the large scale photospheric field.

J.-C. Henoux

H. P. GaIl and E. Sedlayr: 'Effects of Correlated Turbulent Velocities on Photospheric Line Formation', Astron. Astrophys. 36 (1974), 17.

The concept of stochastic turbulent fields is applied to line formation in the solar photosphere. The effect of a finite correlation length on the line profiles, the equivalent widths and the curves of growth are discussed.

J.-C. HeNOUX

R. M. E. Illing, D. A. Landman, and D. L. Mickey: 'Observations of Broad-Band Circular Polarization in Sunspots: Magnetic Field Correspondence', Astron. Astrophys. 37 (1974), 97.

A general rule relating the polarity of broad-band (spectral range: $5250 \AA$ to $5350 \AA$ ) circular polarization fields observed in sunspots to that of the corresponding magnetic fields is presented.

\section{J.-C. Henoux}

S. SAHAL-BrÉchot: 'Role of Collisions in the Polarization Degree of the Forbidden Emission Lines of the Solar Corona, II, Astron. Astrophys. 36 (1974), 355.

The general theory of the polarization of the forbidden lines of the solar corona is reviewed and presented in a simple way. The role of the cascades in the depolarization rate is emphasised, and the polarization degree of the green line is computed by solving the statistical equilibrium equations for the Zeeman sublevels of the system. 
P. LÉNA and Y. VIALA: 'The Thermal Emission of the Dust Corona, during the Eclipse of June 30, 1973', Astron. Astrophys. 37 (1974), 81.

Observations of the F-corona have been made in the $10 \mu \mathrm{m}$ region of the infrared during the eclipse of June 30, 1973. Use of the supersonic aircraft 'Concorde 001' permitted $74 \mathrm{~min}$ of observation during totality and greatly reduced problems due to sky noise. The plane of the ecliptic was scanned over heliocentric distances of from 3 to 19 solar radii off the east limb. Bright features previously observed at shorter wavelengths, notably emission at 4 solar radii are evident on the $10 \mu$ scans, strongly indicating that the radiation is due to thermal emission by dust. Spectra were taken at one region in the ecliptic and tentatively attributed to silicate-type material.

J.-C. Henoux

D. R. Flower and G. Pineau des Forêts: 'On the Analysis of Solar XUV Observations and the Structure of the Chromosphere-Corona Transition Region', Astron. Astrophys. 37 (1974), 297.

The method of analysis of solar XUV line intensities is reconsidered in the light of recent observational determinations of the electron and acoustic pressure in the transition region. The assumptions of constant electron pressure and constant thermal conduction flux in the outer transition region are shown to be incompatible with these observations. A model is derived which is consistent with the available observations and which is characterised by (1) a constant electron density and (2) an electron temperature $\left(T_{e}\right)$ gradient varying as $T_{e}^{-1 / 2}$ in the outer transition region $\left(10^{5} \lesssim T_{e} \lesssim 10^{6} \mathrm{~K}\right)$. (Authors' abstract.)

S. O. KastNer, E. D. Rothe, and W. M. NeuPERT: 'Limb-Brightening Observations from the OSO-7 Satellite. I: Electron Density and Temperature of the Non-Equatorial Corona from EUV Lines', Astron. Astrophys. 37 (1974), 339.

Spectra in the range 190-300 $\AA$ observed by the Goddard spectroheliograph on OSO-7 at several coronal locations beyond the limb are analysed. Preliminary values of electron density are derived. Electron temperature along solar radii at polar angles of $46^{\circ}$ and $8^{\circ}$ are obtained.

J.-C. Henoux

E. FürsT and O. HACHENBERG: 'The Center-to-Limb Variation of the Sun at Centimetric Wavelengths', Astron. Astrophys. 36 (1974), 123.

Observations of the Sun at $5 \mathrm{GHz}, 10.69 \mathrm{GHz}, 17 \mathrm{GHz}$ and $24 \mathrm{GHz}$ are undertaken in order to determine more precisely than before the center-to-limb variation of the brightness temperature. The centerto-limb variation is very small within the frequency range between $10 \mathrm{GHz}$ and $30 \mathrm{GHz}$. However, the observations at $5 \mathrm{GHz}$ indicate a strong limb brightening of approximatively $3000 \mathrm{~K}$ or more at $65^{\circ}$ heliographic longitude. The observations are interpreted by assuming that the lower part of the chromosphere-corona transition region is very inhomogeneous and that the brightness temperature of spicules depends on frequency.

J.-C.HeNouX

B. Møller-Pedersen: 'Solar Noise Storms in the Decametric Wavelength Range', Astron. Astrophys. 37 (1974), 163.

High resolution dynamic spectra of decametric storm activity, obtained during 15 months with the Nançay spectrographs, are described. It is shown that the kind of bursts involved in a decametric storm depends on the longitude of the source. This center-to-limb variation of the nature of the storms might give a clue to the mechanism of emission of the fast decametric bursts. 
J. L. Steinberg, C. Caroubalos, and J. L. Bougeret: 'STEREO-1 Measurements of the Beam Pattern of 169 MHz Type I Bursts on November 18, 1971', Astron. Astrophys. 37 (1974), 109.

The beam pattern of type I bursts is investigated for the first time using simultaneous $169 \mathrm{MHz}$ observations from two different directions: from the Soviet probe Mars-3 and Nançay (France).

J.-C. HENOUX

G. Daigne and B. Møller-Pedersen: 'Characteristic Pairs of Type III Solar Radio Bursts', Astron. Astrophys. 37 (1974), 355.

Observations of type III solar radio bursts appearing in characteristic pairs fundamental-harmonic emissions are reported. Time delay and frequency ratio of the intensity maxima, peak intensities, half power duration and decay time of the intensity time profiles have been measured. The whole set of observations is found not consistent with a strong amplification and an exponentially growing $F$-radiation for a source model with uniform brightness temperature.

J.-C. Henoux 